\title{
Kompetenz-Fundamentalismus und Legitimationsarmut
}

\author{
Fritz Oser \\ Universität Freiburg/Schweiz
}

Es ist zu einer unerträglichen Selbstverständlichkeit geworden, alles, was in der Bildungsforschung publiziert wird, an sogenannte Kompetenzen, die Bildung und Ausbildung bestimmen sollen, zurückzubinden. Typisch etwa: „In dieser Studie stehen Kompetenzen im Unterrichtsfach Deutsch im Fokus, da diese im Rahmen der schulischen Bildung eine besondere Rolle einnehmen: Sprachliche Fähigkeiten stellen die Grundlage für eine aktive und erfolgreiche Teilhabe am gesellschaftlichen Leben dar.“ (Lenski u. a. 2015, auf Artelt u. a. 2002 verweisend) Es geht also um sprachliche Fähigkeiten, und man nennt sie jetzt sprachliche Kompetenzen. Drei Verwendungsformen treten auf:

1. Der Gebrauch des Wortes Kompetenz ist ein Synonym für Fähigkeiten, Wissen, Können, Zielerreichung usw. Es beinhaltet nichts Neues.

2. Es geht um zentrale Unterscheidungen. Z. B. wird angenommen, dass Kompetenzen im beruflichen Bereich notwendige Komplexitäten darstellen und dass sie dort niemals durch schulische Wissensformen abgedeckt werden können. Berufliche Kompetenzen sind mit offenen Handlungsweisen gekoppelt, die oft weniger Wissen beanspruchen, als man Wissen haben muss. Das Handeln im Feld stellt eine Art Validierung der Kompetenz dar.

3. Es wird behauptet, dass die Ausrichtung allen Unterrichts auf Kompetenzen auf allen Stufen eine neue Schulform begründe. Jetzt würde nicht mehr einfach kaltes oder tacit Wissen vermittelt wie früher, sondern es würde kognitive Aktivität stimuliert. Kompetenzorientierung würde als Haltung der Lehrkraft verstanden, die einerseits entscheidend für die Planung, Durchführung und Evaluation des Unterrichts sei (ebd.), andererseits gehe es um Orientierung an den Bildungsstandards. Und manchmal wird Eigenaktivität der Lernenden, entdeckendes Lernen und vor allem zielorientierter Output genannt. Im Schweizer Lehrplan 21, an dem über 140 Personen mitgearbeitet haben, heißt es: „Damit wird signalisiert, dass der Lehrplan nicht bereits erfüllt ist, wenn der im Lehrplan aufgelistete Stoff im Unterricht behandelt wurde, sondern erst dann, wenn die Kinder und Jugendlichen über das nötige Wissen verfügen und dieses auch anwenden können." (Lehrplan 21 2014, o. S.) Es geht also um Wissen, das angewandt werden kann. Man sagt, dass es nicht mehr um Wissensvermittlung, sondern um etwas Neues gehe (vgl. auch Klieme/ Hartig 2008). Oft wird einfach ein fehlender Transfer beklagt.

All das ist erstaunlich, wenn man die Bestrebungen der Curriculumreform, der Unterrichtsqualitätsreform, der Schulklimareform, der pädagogisch-psychologischen Fundierung der Didaktik der letzten Jahrzehnte (s. etwa H. Aebli mit seinem Buchtitel „Denken: Das Ordnen des Tuns“), der „situated learning“Bewegung usw. verfolgt. Dazu eine Situation: Es war meine erste Didaktikstunde am Lehrer- 
seminar Solothurn. „Papi Scholl“, so nannten die Studierenden ihn liebe- und respektvoll, machte uns zuerst eine Unterrichtsstunde vor. Wir saßen im Klassenraum und beobachteten nach Kriteriumsvorlagen. Anschließend gab er Erklärungen, warum er das und jenes in dieser Weise und nicht anders realisiert habe.

Die Stunde lief folgendermaßen ab: Er stellte die Aufgabe, dass ein Maler die Wände der Zimmer eines Hauses mit weißer Farbe ausmalen sollte. Es gab kleine, mittelgroße und große Zimmer, was auf einer Skizze deutlich wurde. Zwei kleine Zimmer kosteten je 100 Fr., zwei mittlere je 200 Fr. und ein großes, das sich auf 300 Fr. belief. Er fragte die Schüler und Schülerinnen, wie man am besten vorgehen müsse, um die Gesamtkosten zu berechnen und ließ sie in Gruppen diskutieren. Beim darauffolgenden Gespräch meinten einige, zuerst seien die Hunderter zusammenzuzählen, andere wollten mit den größten Beträgen beginnen, wiederum andere behaupteten, es spiele keine Rolle. Er bat verschiedene Gruppen an die Wandtafel und gab je die Aufgabe, mit den Hunderten oder den Zweihunderten oder dem Dreihunderter oder nach Zufall zusammenzuzählen, und die Schüler staunten darüber, dass immer der gleiche Betrag herauskam. Er ließ sie diskutieren, warum dies so sei, und sie fanden die Regel, dass die Reihenfolge von addierten Zahlen die Gesamtsumme nie verändert (Erhaltung der Gesamtmenge). „Papi Scholl“ erklärte uns anschließend zwei Grundprinzipien, nämlich a) dass die Lernenden jede Information, die sie aufnehmen, auch zu verarbeiten und zu transformieren, wieder sichtbar zu machen hätten. Er nannte dies Funktionsrhythmus, und er verwies auf Schulreformer wie Karl Stieger und auf die Reichsschulkonferenz. Das zweite Prinzip nannte er Umgang mit Wissen; er erklärte, dass die Schüler und Schülerinnen nicht nur wissen müssten „dass“, sondern auch „wie“ und „warum“. Wir waren damals sehr betroffen von „Papi Scholl“, weil die Kinder der 3. Primarklasse unglaublich aktiv bei der Sache waren, mit- machten, interagierten und offene Blicke hatten. Es war, als ob sie ganz große Erkenntnisse gewonnen hätten.

\section{Das war 1956.}

Blicke ich auf diese und ähnliche Stunden zurück, wird mir bewusst, wie fein der Wechsel zwischen Eigenaktivität und geführter Aktivität war, wie hoch die kommunikative Austauschkultur zwischen Lehrer und Kindern und der Kinder untereinander war, wie subtil Bedingungen zur Entdeckung eines mathematischen $\mathrm{Zu}$ sammenhangs arrangiert wurden, wie gut die Einleitung als Story einer Wohnung, die langsam hässlich und ältlich zu sein schien (Motivation), daherkam und abstrahiert wurde, aber auch wie wichtig das Sichtbarmachen der Resultate der Kinder und die Rückmeldung war. Das waren Momente, wo wir glaubten, dass Lehrersein ein Beruf sei, der Erkenntnis ermögliche, soziale Fähigkeiten stimuliere, interessante Lehr-Lernsituationen schaffe, um der nächsten Generation die Augen für die Großartigkeit und Schönheit der Welt zu öffnen. Ich habe Peter Buser, einem Freund und Mitstudenten, geschrieben, ob er sich an „Papi Scholl“ erinnere. Alle erinnern sich, meinte er. Heute würde man, was hier abgelaufen ist, als Lernkultur bezeichnen. Und der „output“ war eindeutig.

Wer glaubt, mit der Formulierung von vielen Hunderten von Kompetenzen in allen Fächern - wie etwa im Lehrplan 21 - eine solche Schulund Lernkultur zu stimulieren und erreichen zu können, liegt vermutlich falsch. Wenn in der Erziehungsdirektorenkonferenz behauptet wird, früher hätte man nur Wissen vermittelt, heute würde man Kompetenzen anstreben, so ist dies in zwei Richtungen unwahr. Erstens stimmt es nicht, dass früher nur Wissen vermittelt worden wäre (ich glaube, so wäre niemand Erziehungsdirektor geworden), und zweitens stimmt es nicht, dass die heutigen Lehrpersonen so kurzsichtig sind und nur Kompetenzen fördern. 
Abgesehen vom Ansatz 2, den wir oben angedeutet haben, wo es tatsächlich um berufliche Kompetenzen geht, liegt das Problem darin, dass eine wichtige Form des Umgangs mit den Bildungsausrichtungen in eine fatal falsche Richtung gelaufen ist. Als Oser und Oelkers 2001 mit Standards für die Lehrerbildung die Forderung aufstellten, dass für berufliches Können und im Besonderen für Lehrkräfte sogenannte Kompetenzenprofile mit Qualitätsmaßen formuliert, ausgebildet und auf ihre Wirkung hin evaluiert werden sollten und erste empirische Ansätze dazu vorstellten, wurde dieses Konzept ohne Adaptation auf das Lernen der Kinder und Jugendlichen in der Schule übertragen.

Hinter dieser Tendenz gibt es eine Reihe von unausgesprochenen Problemen, die notwendigerweise öffentlich gemacht werden müssen. Eines sei hier genannt: Es besteht darin, dass hinter dem Ansinnen von Kompetenz-Lehrplänen eine falsche Annahme über die Funktion von Wissen, über Wissensaufbau, über Wissensformen und über notwendige basale Gegebenheiten bezüglich der Kultur des Wissens steht. Diese Art der falschen Einschätzung von Wissen führt oft zu Aussagen wie, man würde doch jetzt nicht mehr Wissensvermittlung betreiben, sondern eben Kompetenzen herausbilden. Dieses verächtliche Abwerten von Wissen führt dazu, dass nicht gesehen werden sollte, dass Wissen die Hauptressource für schulisches Lernen darstellt (s. etwa Regressionsanalysen, die zeigen, dass Wissen zentral für berufliches Können ist; so etwa bei Abele u. a. [im Druck], 171ff). Sein Umfang geht immer weit über das hinaus, was eine Kompetenz erfordert. Die einfachste Kompetenz-Sichtbarmachung durch die ärztliche Behandlung eines Patienten, z. B. die Behandlung einer eiternden Wunde, macht dies deutlich: Der Arzt/die Ärztin muss substanziell mehr wissen, als für diesen professionellen Akt benötigt wird. Schüler/ Schülerinnen brauchen Sachwissen, Orientierungswissen, Handlungswissen, strategisches Wissen zu jedem Sachbereich. Kompetenz- orientierung, wie sie von Bildungspolitikern und -planern vorgeschlagen wird, befreit uns nicht davon, solches Wissen sinnvoll, begründet und curricular strukturiert so aufzubauen und dabei altersspezifische Gegebenheiten zu berücksichtigen. Viel von diesem Wissen, auf das wir uns in einer Kultur einlassen, ist nicht zum Gebrauch da, sondern zur Orientierung, etwa biografisches Wissen (wie haben andere Menschen gelebt, wie sind die Dinge geworden?), epistemisches Wissen (was und wie erkennen wir?), ästhetisches Wissen (wie sind Kunstwerke strukturiert, sodass wir sie lieben und mit ihnen leben?). In ihrer Schrift über „knowledge based economy“ spricht die OECD über vier Arten von Wissen, nämlich „Know-what refers to knowledge about,facts “" Sachverhalte, z. B. die Einwohnerzahl der Stadt, in der man lebt, zu kennen, ist keine Schande. „Know-why refers to scientific knowledge of the principles and laws of nature": zu verstehen, warum sich die Erde erwärmt, wäre ein Beispiel. „Know-how refers to skills or the capability to do something": Dies käme am nächsten an das heran, was unter Kompetenz verstanden wird. Und schließlich „... knowwho becomes increasingly important. Knowwho involves information about who knows what and who knows how to do what" (OECD 1996, 12).

Wenn Lehrpersonen sagen, dass die Schüler und Schülerinnen z. B. eine Form des geometrischen Tuns (etwa die Berechnung der Fläche eines Dreiecks) in angewandter Form, etwa in Textbeispielen, zu üben und zu überprüfen hätten, so ist dies jene Aktivität, von der wir meinen, sie führe zum Verstehen und Beherrschen einer Regel. Wenn man zu dem, was bis heute als genau diese Fähigkeit, diese Anwendung zu leisten, genannt wird, jetzt Kompetenz sagt, so ist nichts wirklich gewonnen. Und wenn alles nun so genannt werden muss, damit es berücksichtigt wird, so erinnert dies an Ismen, bei denen alles unter ein und demselben Gesichtspunkt angesehen werden muss. Und wenn 
dies auch für „banale Kompetenzen“ gelten muss, wie etwa „... sich darauf einlassen können, immer wieder neue Bilderbücher, Hörbücher, Hörspiele, Filme anzuschauen, zu lesen, zu hören und darüber zu sprechen", dann versteht man nicht mehr, wo die Wurzeln des Könnens wirklich Wurzeln schlagen. Die Legitimation für diesen Kompetenzformulierungswahn bricht zusammen. Die wohlbegründbare Absicht der Vereinheitlichung der Inhalte darf nicht der Unterkomplexität der Darstellung des unterrichtlichen Prozesses zum Opfer fallen. Zudem macht der Glaube an die Wirkung von Formulierungen den Zustand einer entsprechenden komplexen Verhaltensdynamik nicht besser.

\section{Literatur}

Abele, S.; Behrendt, S.; Weber, W.; Nickolaus, R. (im Druck): Berufsfachliche Kompetenzen von KfzMechatronikern - Messverfahren, Kompetenzdimensionen und erzielte Leistungen (KOKO Kfz). In: Beck, K.; Landenberger, M.; Oser, F. (Hrsg.): Technologiebasierte Kompetenzmessung in der beruflichen Bildung. Bielefeld: Bertelsmann, 171- 203

Aebli, H. (1993): Denken: Das Ordnen des Tuns. 2 Bde. 2. Aufl. Stuttgart: Klett-Cotta

Artelt, C.; Schiefele, U.; Schneider, W.; Stanat, P. (2002): Leseleistungen deutscher Schüler und
Schülerinnen im internationalen Vergleich (PISA). In: Zeitschrift für Erziehungswissenschaft 5, 6-27. http://dx.doi.org/10.1007/s11618-0020002-1

Klieme, E.; Hartig J. (2008): Kompetenzkonzepte in den Sozialwissenschaften und im erziehungswissenschaftlichen Diskurs. In: Prenzel, M.; Goglin, I.; Krüger, H. H. (Hrsg.): Kompetenzdiagnostik. Wiesbaden: VS Verlag für Sozialwissenschaften, 11-29. http://dx.doi.org/10.1007/ 978-3-531-90865-6_2

Lehrplan 21 (2014): Kompetenzorientierung. Online unter: www.lehrplan21.ch/kompetenzorien tierung

Lenski, A. E.; Richter, D.; Pant, A. (2015): Kompetenzorientierung im Unterricht aus der Perspektive von Lehrkräften und Schülerinnen und Schülern. In: Zeitschrift für Pädagogik 61, 712-737

Oser, F.; Oelkers, J. (Hrsg) (2001): Die Wirksamkeit der Lehrerbildungssysteme. Von der Allrounderausbildung zur Ausbildung professioneller Standards. Zürich: Rüegger

OECD (1996): The knowledge-based economy. Paris: Organisation for Economic Co-Operation and Development

\section{Anschrift des Autors}

Prof. em. Dr. Dr. h.c. mult. Fritz Oser

Departement Erziehungswissenschaft Universität Freiburg

$\mathrm{CH}-1700$ Fribourg

fritz.oser@unifr.ch 